\title{
Characterization, Evaluation, and Comparison of Laser-Trimmed Film Resistors
}

\author{
JAIME RAMÍREZ-ANGULO, MEMBER, IEEE, RANDALL L. GEIGER, SENIOR MEMBER, IEEE, \\ AND EDGAR SÁNCHEZ-SINENCIO, SENIOR MEMBER, IEEE
}

\begin{abstract}
Methods for predicting the performance of laser-trimmed film resistors taking into account the properties of the heat-affected zone (HAZ) are discussed. A figure of merit based upon a sensitivity function called $\mathrm{HAZ}$ sensitivity, $S^{H A Z}$, is introduced which is useful for determining aging and temperature effects of an arbitrary film-resistor geometry with an arbitrary trim strategy. $S^{H A Z}$ is also shown to be useful in predicting performance of ratio-matched resistor structures. The proposed technique is incorporated in FIRE, a Fortran program for analyzing arbitrary film structures with a given trim path. Examples using popular resistor geometries and trimming algorithms illustrating the use of the suggested figure of merit are presented. The performances of these structures are compared quantitatively.
\end{abstract}

\section{INTRODUCTION}

L ASER TRIMMING of film resistors is an established process which allows manufacturers to very accurately control resistance values and resistor ratios [1]-[3]. Laser trimming is, however, an expensive processing step which significantly increases the production costs of integrated circuits from both trimming-cost and area-overhead viewpoints. Consequently, it is important to maximize benefits derivable in product performance once the investment in the laser-trimming step is made.

Ideally, nearly infinite resolution of resistance values is possible with appropriate geometrical trim structures using existing commercially available laser-trimming systems. The question of how precisely one can practically expect to control the characteristics of laser-trimmed circuits naturally arises. It is our feeling that the accuracy attainable with laser-trimmed structures should be ultimately determined by the noise floor in a process and that the major reason such precision is not currently realized is due to inadequate characterization of trim structures and the trim processes itself.

When characterizing a given trim structure, three important and interrelated points must be considered. Specifically, these are resistance determination, temperature effects, and aging.

Manuscript received June 10,1986; revised June 20, 1987. This work was supported by Texas Instruments Incorporated.

The authors are with the Department of Electrical Engineering, Texas A\&M University, College Station, TX 77843-3128.

IEEE Log Number 8717253

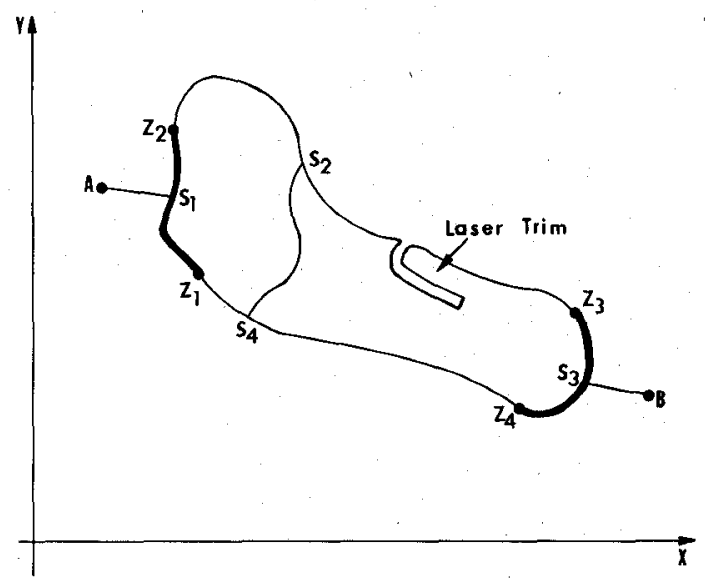

Fig. 1. Arbitrarily shaped laser-trimmed resistor.

An arbitrarily shaped trimmed film resistor is shown in Fig. 1. Perfectly conducting plates are assumed along sides $S_{1}$ and $S_{3}$. Solving for the equivalent resistance between terminals $A$ and $B$ in the case that the sheet resistance is constant over the entire surface typically requires solution of the field equation

$$
\nabla^{2} V=0
$$

with boundary conditions

$$
\begin{aligned}
V_{A} & =0 \\
V_{B} & =\text { constant } \\
\left.\boldsymbol{E} \cdot \boldsymbol{n}(x, y)\right|_{s_{2}} & =0 \\
\left.\boldsymbol{E} \cdot \boldsymbol{n}(x, y)\right|_{s_{4}} & =0
\end{aligned}
$$

and Ohms law

$$
J=\sigma_{n} E
$$

where $\boldsymbol{E}$ is the position-dependent electric field, $\boldsymbol{n}(x, y)$ is a unit vector normal to the boundary at the boundary point $(x, y), V_{A}$ is the potential at node $A, V_{B}$ is the potential at node $B, \sigma_{n}$ is the normalized conductivity which relates to the sheet resistance $R_{\square}$ by the expression

$$
\sigma_{n}=\frac{1}{R_{\square}}
$$




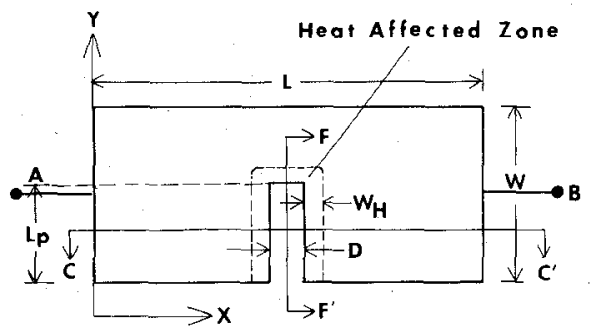

(a)

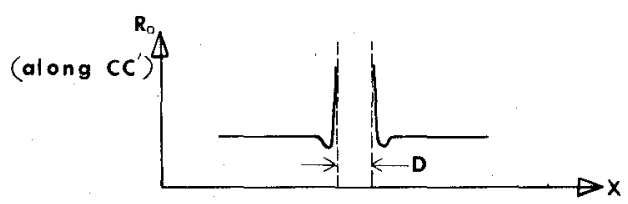

(b)

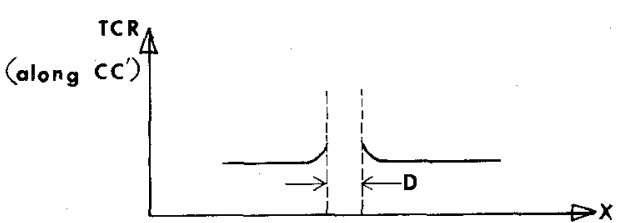

(c)

Fig. 2. Changes in resistivity and TCR in HAZ. (a) Rectangulartrimmed resistor. (b) Sheet resistance (at fixed ambient temperature and current level). (c) TCR.

and $\boldsymbol{J}$ is the current density per unit length. If $\gamma$ is any simple curve between $S_{2}$ and $S_{4}$, the equivalent resistance is obtained from the line integral of $\boldsymbol{J}$ along $\gamma$ as indicated in the expression

$$
R=\frac{V_{B}}{\int_{\gamma} \boldsymbol{J} \cdot \boldsymbol{n}_{1} d l}
$$

where $\boldsymbol{n}_{1}$ is a unit vector normal to $\gamma$.

Unfortunately, the laser trim itself causes heating and melting of the film material near the trim. This heating causes a change in the sheet resistance, the temperature coefficient of the resistance (TCR), and the aging characteristics in the zone adjacent to the trim, henceforth termed the heat-affected zone (HAZ). A trimmed rectangular resistor is shown in Fig. 2 along with the sheet resistance and TCR along the cross section $C-C^{\prime}$. Since the sheet resistance is temperature dependent, the shape of the curve in Fig. 2(b) will change with both ambient temperature and current flow due to self-heating effects. From Fig. 2 it should be apparent that the HAZ is not a well-defined region and the "width of the kerf," $D$, is also not well-defined. As a consequence, the sheet resistance $R_{\square}$ which was assumed constant in the initial field formulation now becomes position, temperature, time, and operating current dependent causing the conductivity to have the same variable dependence

$$
\sigma_{n}(x, y, T, t, I) \text {. }
$$

The last three parameters in this expression do not complicate the solution of the field equation but merely affect the numerical value of the solution. The spatially dependent parameters, however, cause significant complication of the field equation since the gradient of $\sigma_{n}$ is not zero. As a consequence, Poisson's equation (1) must be replaced by

$$
\sigma_{n}(x, y) \nabla^{2} V+\nabla V \cdot \nabla \sigma_{n}(x, y)=0
$$

The remainder of (2)-(9) is held unchanged.

At this point one might naturally ask whether it is necessary to develop analytic tools for characterizing trimmed film structures since current laser-trimming technology provides nearly infinite trim resolution and since one will ultimately be forced to utilize structures which contain the HAZ after trimming anyway. It is, however, generally agreed that some trim geometries are more stable after trim than others. The lack of good analytical tools has impeded the evolution of optimal geometric structures for laser-trimming applications.

A method to characterize and compare laser-trimmed film resistors is presented. A computer program for analyzing FIlm REsistors of arbitrary geometries and a positiondependent sheet resistance, which essentially solve the field equations (2)-(10), is discussed. This program, called FIRE, uses a simple numerical relaxation technique for determining current and voltage distributions throughout the film resistor. FIRE also obtains power distributions and has provisions for automatic characterization of the $\mathrm{HAZ}$ in any specified trim path. Beyond serving as a tool for characterizing film resistors, the program should prove useful in determining optimal film resistor geometries and in establishing good trim algorithms.

A figure of merit, $S^{H A Z}$, is also introduced which is useful for predicting temperature effects and aging characteristics of arbitrarily shaped laser-trimmed film resistors. Such a figure is needed for designing trim structures with reduced sensitivity to the trim. Applications of the figure of merit in characterizing the performance of some commonly used film resistors are discussed. $S^{H A Z}$ is also calculated directly in FIRE.

\section{Heat-Affected Zone Effects}

Trimmed film resistors (TFR's) show different performance with respect to aging and temperature variations than untrimmed resistors. In addition, the performance of TFR's is both trim path and geometry dependent [4]-[6]. It is desirable to keep the absolute and/or relative resistance changes due to aging and/or temperature variations as low as practically possible. In particular, these changes must stay within a predetermined performance window to maintain system specifications throughout the intended lifetime of the product.

A laser beam is commonly used to trim film resistors. During the trimming process, a region along the edge of the resultant laser cut is nonuniformly heated to very high temperatures by the laser beam but the temperature rise is not enough to vaporize the material. Part of the nonuniformity of the heating in the HAZ is due to the fact that 


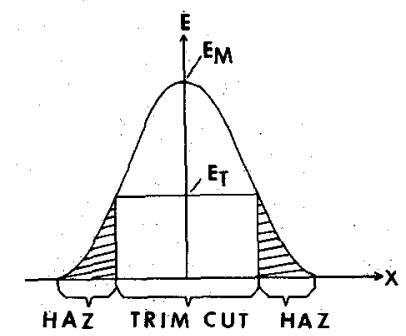

Fig. 3. Spatial laser-beam energy distribution, where $E_{M}$ and $E_{T}$ denote the maximum laser-beam energy and the vaporization threshold energy, respectively.

the energy distribution in the laser beam is not uniform (see Fig. 3). The heat transfer characteristics of the film material and the bulk substrate also affect the temperature space-time profile of the HAZ. After cooling, this "heataffected zone" is characterized by physical properties which differ from the untrimmed portions of the resistor. Specifically, changes occur in the sheet resistance, temperature coefficient, and the aging behavior, as indicated in Fig. 2. Neither the width of the HAZ $W_{H}$ nor the width of the laser kerf $D$ as depicted in Fig. 2 is well defined. The changes in the sheet resistance from the nominal untrimmed value are most significant in a region of width $W_{H}$ adjacent to the kerf as indicated in Fig. 2. From an area viewpoint, the $\mathrm{HAZ}$ comprises only a few percent of the total film-resistor area after trimming; thus the characteristics of the HAZ are of concern primarily in high-precision applications.

Of even more interest than the percent of the total film-resistor area which lies inside the HAZ is the powerdensity-weighted area which lies inside the HAZ. It will be shown later that a figure of merit based upon the power density in the HAZ is useful for predicting performance of trimmed-resistor structures.

There are two primary reasons why the power dissipated in the HAZ plays a major role in the performance of film resistors. First, most trim strategies indice "current crowding" near the tip of the trim. This can be seen easily from the plunge-trim ${ }^{1}$ structure of Fig. 2(a). Assume initially that the sheet resistance in the HAZ is the same as that of the untrimmed film and that a constant current $I$ is applied to the resistor. A plot of the current density (current per unit length) along cross section $F-F^{\prime}$ is shown in Fig. 4 for varying plunge depths. It is apparent from the peaks in these curves that current is crowding near the tip of the trim.

The second reason the power density is of concern is due to the additional current crowding induced due to a decrease in the resistivity in the HAZ, which is characteristic of laser trims [6] as was shown in Fig. 2(b). Due to the decrease in resistivity, the current is "funneled," additionally, along the edge of the trim through the lower resistivity HAZ into the tip itself. The current density along $F-F^{\prime}$ is shown in Fig. 5 under the assumption that the

\footnotetext{
${ }^{1}$ The term plunge cut is often used to denote a single linear trim which is perpendicular to the geometrical boundary where the cut originates.
}

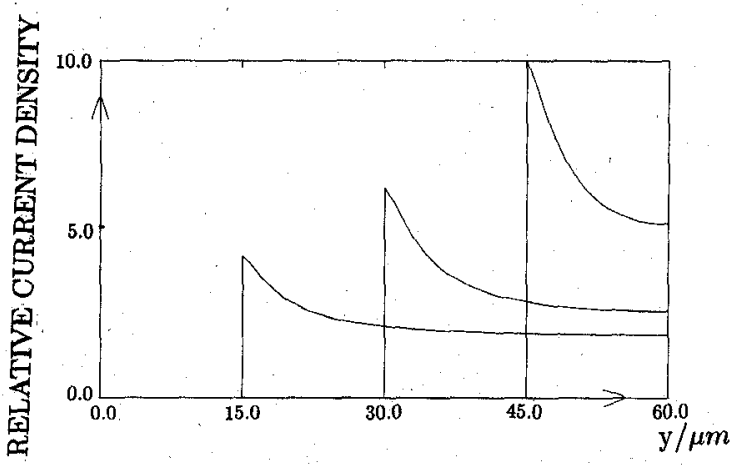

Fig. 4. Current density along $F-F^{\prime}$ for various plunge depths with constant sheet resistance for a bar resistor with length $L=120 \mu \mathrm{m}$, width $W=60 \mu \mathrm{m}$, and width of laser cut $D=5 \mu \mathrm{m}$.

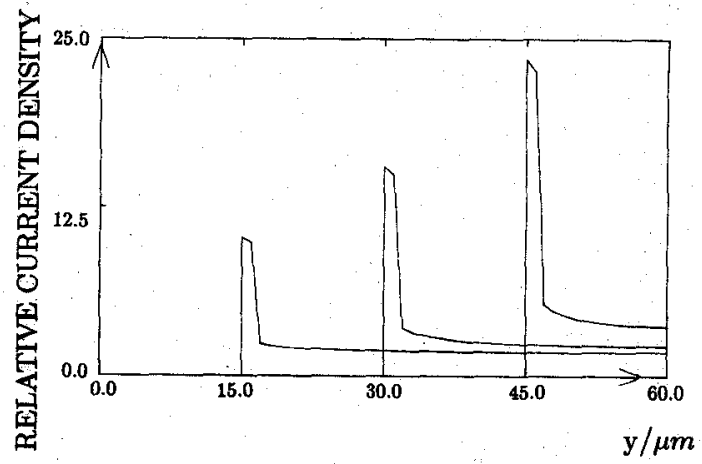

Fig. 5. Current density along $F-F^{\prime}$ for various plunge depths with two-zone model of sheet resistance.

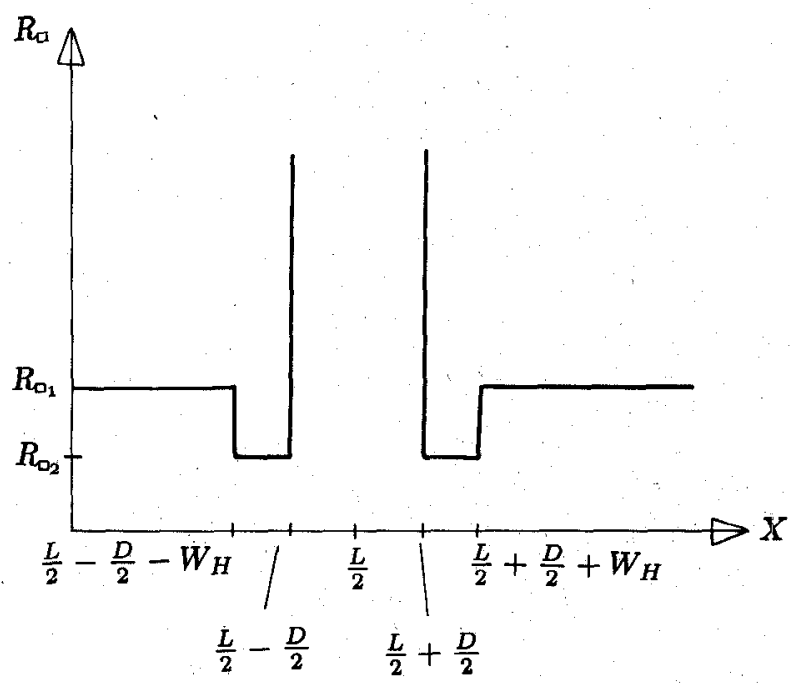

Fig. 6. Two-zone model for resistivity of laser kerf (see Fig. 3(a) and (b)).

resistivity can be approximated by the two-zone model of Fig. 6 with $W_{H}=2 \mu \mathrm{m}$ and $R_{\square 2}=R_{\square 1} / 4$, where $R_{\square 1}$ and $R_{\square 2}$ are the sheet resistances in the two zones shown in Fig. 6. The significant increase in current density in the region where current crowding is causing problems already due to a decrease in the sheet resistance in the HAZ is apparent.

A third factor which deserves mention is the self-heating in the HAZ due to the current crowding. This self-heating can make the resistance somewhat current level dependent 


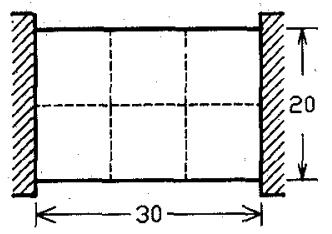

(a)

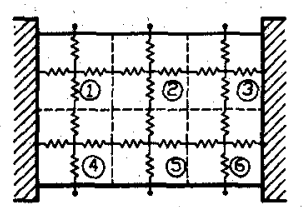

(c)

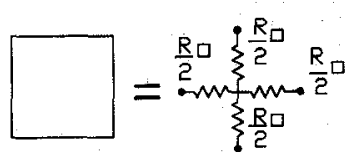

(b)

(1) (2) (3)

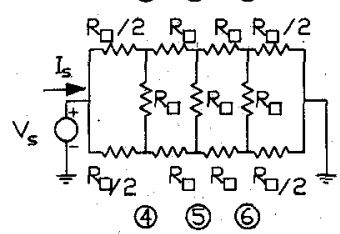

(d)
Fig. 7. Derivation of an equivalent circuit for a homogeneous film resistor. (a) Homogeneous film resistor with aspect ratio $3: 2$ divided into six squares. (b) Equivalent circuit for a square element. (c) Circuit representation of film resistor with 24 resistors. (d) Simplified circuit representation.

(i.e., nonlinear) and may be a major failure mechanism under large currents. It should be apparent that if the self-heating damages the film, the kerf depth will tend to grow and the degree of self-heating will increase with the kerf depth.

\section{Equivalent Circuit Representation of A FILM RESISTOR}

Methods for solving the two-dimensional stationary field problem associated with the analysis of a film resistor are available [7]. One of these methods consists of subdividing the resistor structure into a large number of small elements (small enough to describe the resistor geometry with sufficient resolution). With this approach, a resistive circuit representation of the film resistor is obtained by replacing each square by the equivalent circuit shown in Fig. 7(b). This analysis technique leads to an equivalent resistive circuit which contains a large number of nodes. For a fixed excitation, each nodal voltage approximately represents the potential at the corresponding position of each square. An iterative relaxation algorithm can be used to rapidly obtain the nodal voltage in the resistive network. Once the potential distribution of the TFR has been determined, the calculation of its overall resistance $R$ can be easily accomplished by using the expression

$$
R=\frac{V_{S}}{I_{S}}
$$

where $I_{S}$ is the current supplied by the fixed voltage source $V_{S}$ (Fig. $7(\mathrm{~d})$ ). The relaxation algorithm is equally well suited for fixed sheet resistance and spatially dependent sheet resistance analyses.

The program FIRE was written to find the nodal voltages at points inside the resistor. FIRE uses a numerical over-relaxation algorithm [7]. The film resistor is initially decomposed into a finite number $M$ of orthogonally aligned

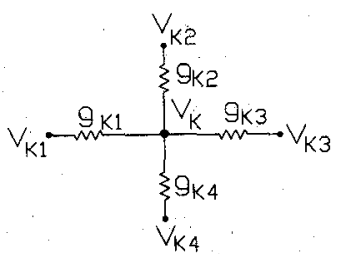

Fig. 8. Lumped-network model of elemental square.

elemental squares. Each square is decomposed into the star connection of four resistors as shown in Fig. 7(b) in which $R_{\square}$ represents the average position-dependent sheet resistance of the elemental square. The $M$ squares are arbitrarily rank ordered. At the $i$ th step in the iterative relaxation algorithm, the nodal voltage at the center of each internal square is updated in accord to the rank ordering at each step by the algorithm

$$
V_{k}^{i}=\frac{\sum_{l=1}^{4} g_{k l} V_{k l}^{x l}}{g_{k 1}+g_{k 2}+g_{k 3}+g_{k 4}}, \quad 1 \leq k \leq M
$$

where $k_{1}-k_{4}$ (see Fig. 8) denote the four nodes adjacent to node $k, g_{k l}$ denotes the conductance between the nodes $k$ and $k l$ for $l=1, \cdots, 4$, and the iterative superscripts $x l$ are defined by

$$
x l= \begin{cases}i, & k l<k \\ i-1, & k l>k\end{cases}
$$

for $1 \leq l \leq 4$.

Outputs from FIRE are the effective resistance of the resistor and plots showing the potential, current, and power density distribution throughout the structure. FIRE differs from other programs [9]-[13] for analyzing film resistors in the following ways:

1) the program works for nonhomogeneous as well as homogeneous films;

2) the program is structured to allow user-enterable trim paths with user-enterable characterization of the HAZ surrounding the trim path; and

3) the program calculates a figure of merit which is useful for the evaluation and comparison of trimmed film structures.

\section{Derivation of a Figure of Merit for FILM-RESISTOR EVALUATION}

\section{A. Sensitivity of Resistive Networks to Individual Elements}

The effects of a single resistor on the overall resistance of a resistance network are investigated in this section. Consider initially the totally resistive one-port network $N K$ excited with a dc voltage source $V_{S}$ as shown in Fig. 9. Assume that $N K$ is comprised of $N$ resistors. It is well known [8] that the partial derivative of the port input impedance $R_{\text {in }}$ of a resistive network with respect to variations in one of its elements $R_{x}$ is given by the 


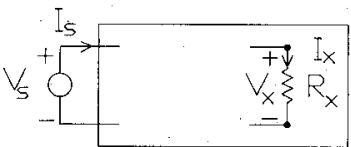

Fig. 9. Resistive network $N K$ representing a film resistor as a one-port resistive network.

expression

$$
\frac{\partial \dot{R}_{\text {in }}}{\partial R_{x}}=\frac{I_{x}^{2}}{I_{s}^{2}}
$$

where $I_{s}$ and $I_{x}$ are the current entering into the port and the branch current in the element $R_{x}$, respectively. Defining the sensitivity of $R_{\text {in }}$ with respect to $R_{x}$ by the standard expression

$$
S_{R_{x}}^{R_{\text {in }}}=\left(\frac{\partial R_{\text {in }}}{\partial R_{x}}\right) \frac{R_{x}}{R_{\text {in }}}
$$

it follows that

$$
S_{R_{x}}^{R_{\text {in }}}=\frac{P_{x}}{P_{\text {in }}}
$$

where $P_{\text {in }}$ and $P_{x}$ are the input power and the power dissipated in $R_{x}$, respectively. This result is very useful in characterizing film resistors.

\section{B. Effects of External Parameters on the Performance of Film Resistors}

Consider now the change in a resistor $R$ with respect to a parameter $y$ such as temperature or aging. If we define a sensitivity function with respect to the parameter $y$ by

$$
S_{y}^{R}=\left(\frac{\partial R}{\partial y}\right) \frac{y}{R}
$$

it follows that fractional changes in $R$ due to changes in $y$ can be approximated by

$$
\left.\left.\frac{\Delta R}{R}\right|_{y=y_{0}} \simeq\left(\frac{y-y_{0}}{y}\right) S_{y}^{R}\right|_{y=y_{0}}
$$

where $y_{0}$ is the nominal value of $y$. If $y$ represents temperature, then conveniently $y_{0}$ would equal the temperature at trim and $S_{y}^{R}$ would represent the temperature coefficient of $R$. If $y$ represents time (aging), then $y_{0}$ would conveniently represent the date of the trim and $S_{y}^{R}$. the "aging coefficient" of $R$.

It should be apparent from (17) that $S_{y}^{R}$ serves as a figure of merit for evaluating the performance of film resistors and should be made as small as possible for trim stability. Consider now the input impedance of the network $N K$ of Fig. 9:

$$
R_{\text {in }}\left(R_{1}(y), R_{2}(y), \cdots, R_{N}(y)\right)
$$

It follows that

$$
\frac{\partial R_{\text {in }}}{\partial y}=\sum_{k=1}^{N} \frac{\partial R_{\text {in }}}{\partial R_{k}} \frac{\partial R_{k}}{\partial y} .
$$

From (14) and (15)

$$
\frac{\partial R_{\text {in }}}{\partial R_{k}}=\frac{P_{k}}{P_{\text {in }}} \frac{R_{\text {in }}}{R_{k}} .
$$

Hence from (16), (19), and (20)

$$
S_{y}^{R_{\text {in }}}=\frac{1}{P_{\text {in }}} \sum_{k=1}^{N} P_{k} S_{y}^{R_{k}} .
$$

This general expression shows the contribution towards the total sensitivity of each element which comprises $R_{\text {in }}$ and serves as a figure of merit for characterizing the performance of a TFR.

\section{Performance of Trimmed Film Resistors}

Since the electrical characteristics of the $\mathrm{HAZ}$ differ from those of the unaffected film, it is necessary to predict the effects of these differences on film-resistor performance. The effects of the entire $\mathrm{HAZ}$ are investigated in this section.

Assume that a film network is decomposed into a lumped approximating network of $N$ geometrically identical resistors as indicated in the previous section. The resistor $R_{k}$ is position dependent. Its value is equal to the average sheet resistance at the location of resistor $R_{k}$ :

$$
R_{k}=R_{\square}(k) \text {. }
$$

The sensitivity of $R_{\text {in }}$ can be expressed in terms of the sheet resistance by

$$
S_{y}^{R_{\text {in }}}=\frac{1}{P_{\text {in }}} \sum_{k=1}^{N} P_{k} \frac{y}{R_{\square}(k)} \frac{\partial R_{\square}(k)}{\partial y} .
$$

Assume that after trim; the film resistor can be decomposed into a zone that was affected by heating, termed the $\mathrm{HAZ}$, and an unaffected zone (the complement of HAZ, $\overline{\mathrm{HAZ}}$, in the geometrical sense relative to the trimmed resistor geometry). From (23), it can be observed that the sensitivity or $R_{\text {in }}$ to the parameter $y$ is the sum over all $N$ resistors of the individual power-weighted sensitivities. It is useful to define the sensitivity of $R_{\text {in }}$ to the parameter $y$ in a subregion by restricting the sum in (23) to the subregion. Since the HAZ is a subregion it follows that the sensitivity to the HAZ is expressed as

$$
S_{H A Z, y}^{R_{\text {in }}}=\frac{1}{P_{\text {in } R_{\square}}} \sum_{(k) \in H A Z} P_{k}\left(\frac{y}{R_{\square}(k)} \frac{\partial R_{\square}(k)}{\partial y}\right) .
$$

This serves as a figure of merit for characterizing the HAZ-related performance of any trimmed film resistor.

Observe from (24) that: a) the power dissipated in the HAZ directly affects the sensitivity; b) current crowding, 
which was previously shown to force additional current into the HAZ with typical trim geometries, further increases $S_{H A Z, y}^{R_{n}} ;$ c) the characteristics of the film in the HAZ, specifically the time and temperature characteristics of the sheet resistance, are of major concern.

In what follows, we will use a two-zone model to characterize a trimmed resistor, i.e.,

$$
R_{\square}=\left\{\begin{array}{ll}
R_{\square 1} & \text { film material outside } \mathrm{HAZ} \\
R_{\square 2} & \text { film material inside HAZ }
\end{array} .\right.
$$

$R_{\square 1}$ and $R_{\square 2}$ are assumed to characterize two geometrically homogeneous regions. (The justification for the twozone model is given later.) $R_{\square 1}$ and $R_{\square 2}$ may, however, have different dependence upon the parameter $y$. This characterization was depicted graphically in Fig. 6. With this simplification, the sensitivity in (24) simplifies to a quantity that can be readily computed

$$
S_{H A Z, y}^{R_{\mathrm{n}}} \simeq\left(\frac{y}{R_{\square 2}}\right) \frac{\partial R_{\square 2}}{\partial y}\left(\frac{1}{P_{\mathrm{In}}} \sum_{R_{k} \in H A Z} P_{k}\right) .
$$

If one compares two trim geometries on a common film, the coefficient

$$
\left(\frac{y}{R_{\square 2}}\right) \frac{\partial R_{\square 2}}{\partial y}
$$

in (26) will remain invariant. Hence, the sensitivity of the two structures varies with the summed power dissipation in the HAZ which serves as a very simple figure of merit for comparing trim structures and trim algorithms. We define the HAZ sensitivity $S^{H A Z}$ by

$$
S^{H A Z}=\frac{1}{P_{\text {in }}} \sum_{R_{\square}(k) \in H A Z} P_{k}=\frac{P_{H A Z}}{P_{\text {in }}}
$$

where $P_{k}$ is the power dissipation of the resistor $R_{k}$ and $P_{H A Z}$ is the total power dissipated in the HAZ.

$S^{H A Z}$ serves as a practical figure of merit for characterizing the effects of the HAZ. From (23) it can be shown that

$$
S_{\gamma}^{R_{\mathrm{in}}}=S^{H A Z} \frac{y}{R_{\square 2}} \frac{\partial R_{\square 2}}{\partial y}+\left(1-S^{H A Z}\right) \frac{y}{R_{\square 1}} \frac{\partial R_{\square 1}}{\partial y} .
$$

The total percent change in $R_{\text {in }}$ can be readily obtained. It follows from (27) and (28) that

$$
\frac{\Delta R_{\mathrm{in}}}{R_{\mathrm{in}}} \simeq \frac{\Delta R_{\square 1}}{R_{\square 1}}+S^{H A Z}\left[\frac{\Delta R_{\square 2}}{R_{\square 2}}-\frac{\Delta R_{\square 1}}{R_{\square 1}}\right] .
$$

Equation (29a) can also be expressed in terms of environmental changes $y-y_{0}$ as follows:

$$
\begin{aligned}
& \frac{\Delta R_{\text {in }}}{R_{\text {in }}} \simeq \frac{\left(y-y_{0}\right)}{R_{\square 1}} \frac{\partial R_{\square 1}}{\partial y} \\
& +S^{H A Z}\left[\left(y-y_{0}\right)\left(\frac{1}{R_{\square 1}} \frac{\partial R_{\square 1}}{\partial y}\right)\left(\frac{\frac{1}{R_{\square 2}} \frac{\partial R_{\square 2}}{\partial y}}{\frac{1}{R_{\square 1}} \frac{\partial R_{\square 1}}{\partial y}}-1\right)\right] .
\end{aligned}
$$

In (29) the first term in the right-hand side represents a common process-dependent change for all film resistors, trimmed or untrimmed, on a common film. The significance of the remaining term is discussed in the following section.

\section{Effects of the HAZ on the Performance of Film Resistors}

The overall error associated with laser trimming which has been neglected in most other analyses will now be determined. To identify these neglected errors, assume that the laser trim has infinite precision at the trim time and temperature and that $S_{y} R_{\text {tn }}$ has been used to predict performance under the assumption that the entire trimmed resistor has been characterized by $R_{\square 1}$ of (25). This assumption may be rationalized since the characteristics of the HAZ are not well-known and since the amount of trimming required of a given resistor is not known prior to trimming. Define the error associated with neglecting the characteristics of the HAZ by the expression

$$
E(y)=\frac{R_{\mathrm{mn}}-R_{\mathrm{in}, R_{\mathrm{al}}}}{R_{\mathrm{in}}}
$$

where $R_{1 \mathrm{n}, R_{\square 1}}$ is the approximation of $R_{\mathrm{nn}}$ obtained by neglecting the change in film characteristics in the HAZ. From (17)

$$
R_{\mathrm{in}} \simeq R_{\mathrm{in}}\left(y_{0}\right)\left[1+\left.\frac{\left(y-y_{0}\right)}{y} S_{y}^{R_{\mathrm{m}}}\right|_{y=y_{0}}\right] .
$$

From (26) and (28)-(30), it now follows that

$$
E(y) \simeq S^{H A Z}\left[\left(y-y_{0}\right)\left(\frac{1}{R_{\square 1}} \frac{\partial R_{\square 1}}{\partial y}\right)\left(\frac{\frac{1}{R_{\square 2}} \frac{\partial R_{\square 2}}{\partial y}}{\frac{1}{R_{\square 1}} \frac{\partial R_{\square 1}}{\partial y}}-1\right)\right] .
$$

It should be observed that $S^{H A Z}$ serves as a practical figure of merit for characterizing the effects of the HAZ. It is useful for comparing film geometries as well as trimming algorithms since all terms in (32) except $S^{H A Z}$ are determined by the process and environment and hence are not controllable by the designer.

To determine the relative significance of the error associated with neglecting the HAZ, consider a numerical example where the variable $y$ is temperature and $T-T_{0}=$ $40^{\circ} \mathrm{C}$. Assume $S^{H A Z}=10$ percent and

$$
\begin{aligned}
& \frac{1}{R_{\square 1}} \frac{\partial R_{\square 1}}{\partial T}=100 \mathrm{ppm} /{ }^{\circ} \mathrm{C} \\
& \frac{1}{R_{\square 2}} \frac{\partial R_{\square 2}}{\partial T}=200 \mathrm{ppm} /{ }^{\circ} \mathrm{C} .
\end{aligned}
$$

It follows from (32) that $E(t) \simeq 0.04$ percent. This becomes significant at the 11-bit level. 
It should be emphasized that $E(y)$ in (32) represents only the incremental error associated with neglecting the HAZ. The overall error is larger and it is given by (29).

\section{E. Ratio-Matching Performance of Trimmed Film Resistors}

Of even more practical concern in many applications is the ratio-matching accuracy maintainable over time and temperature with laser-trimmed resistors. Assume that two resistors $R_{A}$ and $R_{B}$ are both trimmed to values $R_{A T}$ and $R_{B T}$, so that the ratio at trim, $R_{A T} / R_{B T}$, is $r_{0}$. Unfortunately, the ratio $r$ does not remain constant with time and/or temperature. Hence, if we consider a single parameter dependence $y$ we obtain

$$
r(y)=\frac{R_{A T}(y)}{R_{B T}(y)} .
$$

Define

$$
S_{y}^{r}=\left(\frac{\partial r}{\partial y}\right) \frac{y}{r}
$$

Let $S_{A}^{H A Z}$ and $S_{B}^{H A Z}$ denote the fractional power dissipation (as defined by (27)) in $H A Z A$ and $H A Z B$, respectively. Assume both resistors are made of the same film material and characterized by the two-zone model with

$$
\begin{aligned}
& R_{\square 1 A}=R_{\square 1 B}=R_{\square 1} \\
& R_{\square 2 A}=R_{\square 2 B}=R_{\square 2} .
\end{aligned}
$$

A standard sensitivity analysis shows that

$$
\begin{aligned}
& \frac{\Delta r}{r} \simeq\left(S_{A}^{H A Z}-S_{B}^{H A Z}\right) \\
& \cdot\left[\left(y-y_{0}\right)\left(\frac{1}{R_{\square 1}} \frac{\partial R_{\square 1}}{\partial y}\right)\left(\frac{\frac{1}{R_{\square 2}} \frac{\partial R_{\square 2}}{\partial y}}{\frac{1}{R_{\square 1}} \frac{\partial R_{\square 1}}{\partial y}}-1\right)\right]
\end{aligned}
$$

This equation is useful for predicting ratio accuracy. It should be noted that the designer has control of only the term $S_{A}^{H A Z}-S_{B}^{H A Z}$. This can be minimized either by matching the two sensitivities or by making both simultaneously small. Although both approaches should be implemented simultaneously, processing variations ultimately limit the matching of these terms. Clever geometries and trim algorithms can be used to reduce the sensitivities themselves. An illustrated numerical example is given in Section V.

\section{F. Suitability of Two-Zone Model for Characterization of Laser-Trimmed Films}

Referring back to Fig. 2(b) and Fig. 6, two questions naturally arise. First, is the two-zone model of sheet resistance, as depicted in Fig. 6, justifiable for characterizing laser-trimmed film resistors? Second, if justifiable, how is

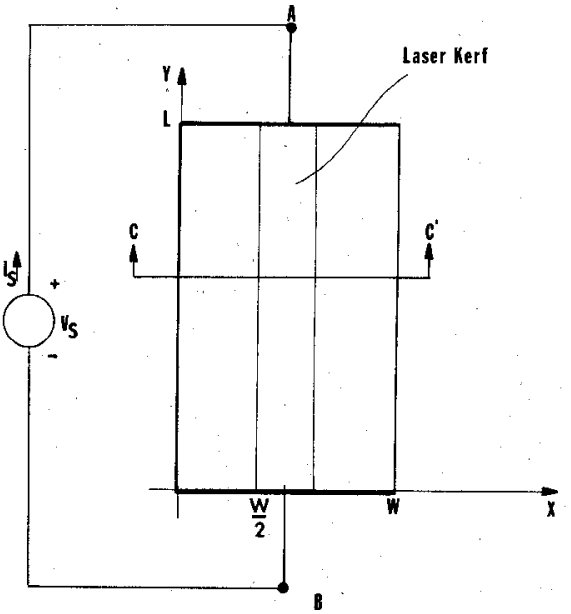

Fig. 10. Laser-kerf characterization structure.

the width of the HAZ selected? We will attempt to answer both questions simultaneously.

To answer these questions, consider the geometrical film resistor structure of Fig. 10 where it is assumed that a uniform vertical laser trim has been made in a homogeneous film. The sheet resistance along cross section $C-C^{\prime}$ will assume a functional form similar to that shown in Fig. 2(b).

If we assume a constant voltage $V_{s}$ is applied to the ends of the film resistor as shown in the figure, it can be concluded, by symmetry, that the electric field is constant throughout the structure and oriented in the $y$ direction with amplitude $V_{s} / L$. Also, by symmetry, the conductivity $\sigma_{n}$ displays a horizontal dependence but no vertical dependence. Hence the current density $\boldsymbol{J}$ is also directed in the vertical direction throughout the structure and is given by

$$
\boldsymbol{J}=\sigma_{n}(x) \cdot \boldsymbol{E} .
$$

Define the curve $\gamma$ to be any horizontal line of length $W$ which bisects the film resistor as shown in the figure. From (6) and (8)

$$
\begin{gathered}
R=\frac{V_{s}}{\int_{\gamma} \sigma_{n}(x) \cdot \boldsymbol{E} \cdot d \boldsymbol{l}}=\frac{V_{s}}{\frac{V_{s}}{L} \int_{x=0}^{W} \sigma_{n}(x) d x} \\
R=\frac{L}{\int_{x=0}^{W} \sigma_{n}(x) d x}
\end{gathered}
$$

which, in terms of the sheet resistance, becomes

$$
R=\frac{L}{\int_{x=0}^{W} \frac{1}{R_{\square(x)}} d x} .
$$

From (39) it should be apparent that it is the integral of $R_{\square}^{-1}(x)$ rather than $R_{\square}^{-1}(x)$ itself which determines the resistance. It can thus be concluded that if one is working with the geometries shown in Fig. 10, it is not necessary to know the shape of $R_{\square}(x)$, only the integral is needed. This 


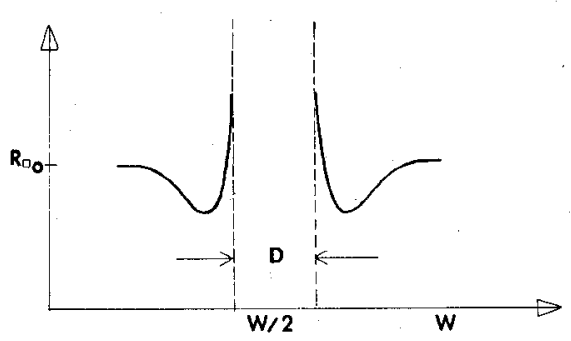

(a)

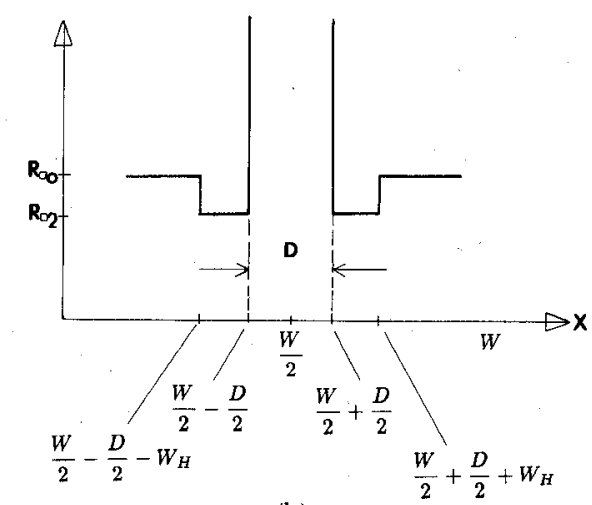

(b)

Fig. 11. Characterization of the sheet resistance of a laser kerf. (a) Typical sheet resistance. (b) Two-zone model.

integral can be measured by fabricating and trimming the structure of Fig. 10, measuring $L$ and $R$ and solving for the integral via (39).

In the two-zone model of Fig. 11(b), the designer can randomly pick any two of the quantities $D, W_{H}$, and $R_{\square 2}$. These quantities are related by the expression

$$
R_{\square 2}=\frac{2 W_{H}}{\frac{W}{R_{\square A}}-\frac{\left(W-D-2 W_{H}\right)}{R_{\square 1}}}
$$

where $R_{\square 1}$ corresponds to the sheet resistance of the material away from the kerf ( $R_{\square 0}$ in Fig. 11(b)) and $R_{\square A}$ is the average sheet resistance along $C-C^{\prime}$, which is defined by

$$
R_{\square A}=\frac{W}{\int_{x=0}^{W} \frac{1}{R_{\square(x)}} d x} .
$$

The model offers the advantage in that the TCR can also be modeled with the same two-zone decomposition in a $W$-independent manner. To see this, observe that the two-zone model is equivalent to decomposing the trimmed resistor into two shunt lumped resistors $R_{1}$ and $R_{2}$, where

$$
R_{1}=R_{\square 0}\left(\frac{L}{W-D-2 W_{H}}\right)
$$

and

$$
R_{2}=\frac{R_{\square 2} L}{2 W_{H}}
$$

Defining the TCR of any resistor by the expression

$$
\mathrm{TCR}=\frac{1}{R} \frac{d R}{d T}
$$

it follows that the TCR of $R_{1} \| R_{2}$, which represents the TCR of the film resistor and a quantity which can be measured, is given by

$$
\mathrm{TCR}=\frac{R_{1}}{R_{1}+R_{2}} \mathrm{TCR}_{2}+\frac{R_{2}}{R_{1}+R_{2}} \mathrm{TCR}_{1}
$$

Hence, if we let $\mathrm{TCR}_{1}$ be the TCR of the untrimmed film and TCR in (45) be the measured TCR of the test structure, then this equation can be solved for an equivalent $W$-independent TCR $\mathrm{T}_{2}$ of the region characterized by $R_{\square 2}$. The values $\mathrm{TCR}_{1}$ and $\mathrm{TCR}_{2}$ can be used to determine $\Delta R_{\square 1}$ and $\Delta R_{\square 2}$ to characterize the temperature performance of an arbitrarily shaped, arbitrarily trimmed resistor using (29). An analogous derivation can be carried out to determine the aging performance of a TFR.

It has been shown that this two-zone model is sufficient for characterizing the laser kerf from both a sheet resistance and TCR viewpoint in a $W$-independent manner. As far as choosing the parameters in the model, reasonable results have been obtained by selecting the kerf width $D$ to be either the laser spot size or the geometric kerf width as determined from a microscope. The width of the HAZ $W_{H}$ has been assumed to be $D / 2$. None of these parameters is critical provided the trim structure is sufficiently large and the trim path does not create any very narrow regions of untrimmed material. When the dimensions of the untrimmed material, after trimming, approach those of the laser kerf width, the two-zone model will not be adequate.

\section{EXAMPLES}

Examples are presented in this section which illustrate the use of $S^{H A Z}$ for evaluating the effects of trimming on the performance of TFR's. The program FIRE was used in all simulations.

\section{A. Comparison of Performance of Trimmed Structures}

A comparison of several different trim algorithms will be considered in this section. This comparison will be restricted to a bar structure that has a $2: 1$ aspect ratio. Three different trim strategies for this structure will be compared. These are, specifically, a symmetric plunge, an $L$ cut, and a multiple plunge strategy. For comparison purposes, all resistors in this simulation were arbitrarily trimmed to 200 percent of their initial value with a square $5 \mu \mathrm{m}$ on a side "laser" spot. The FIRE simulation was based upon decomposition of the film into 800 squares. The trim path and relative power densities for each of these trim algorithms are shown in Fig. 12 assuming the same sheet resistance for the $\mathrm{HAZ}$ and for the untrimmed 


\section{Fractional Power Dissipated in $\mathrm{HAZ}=0.1515$}

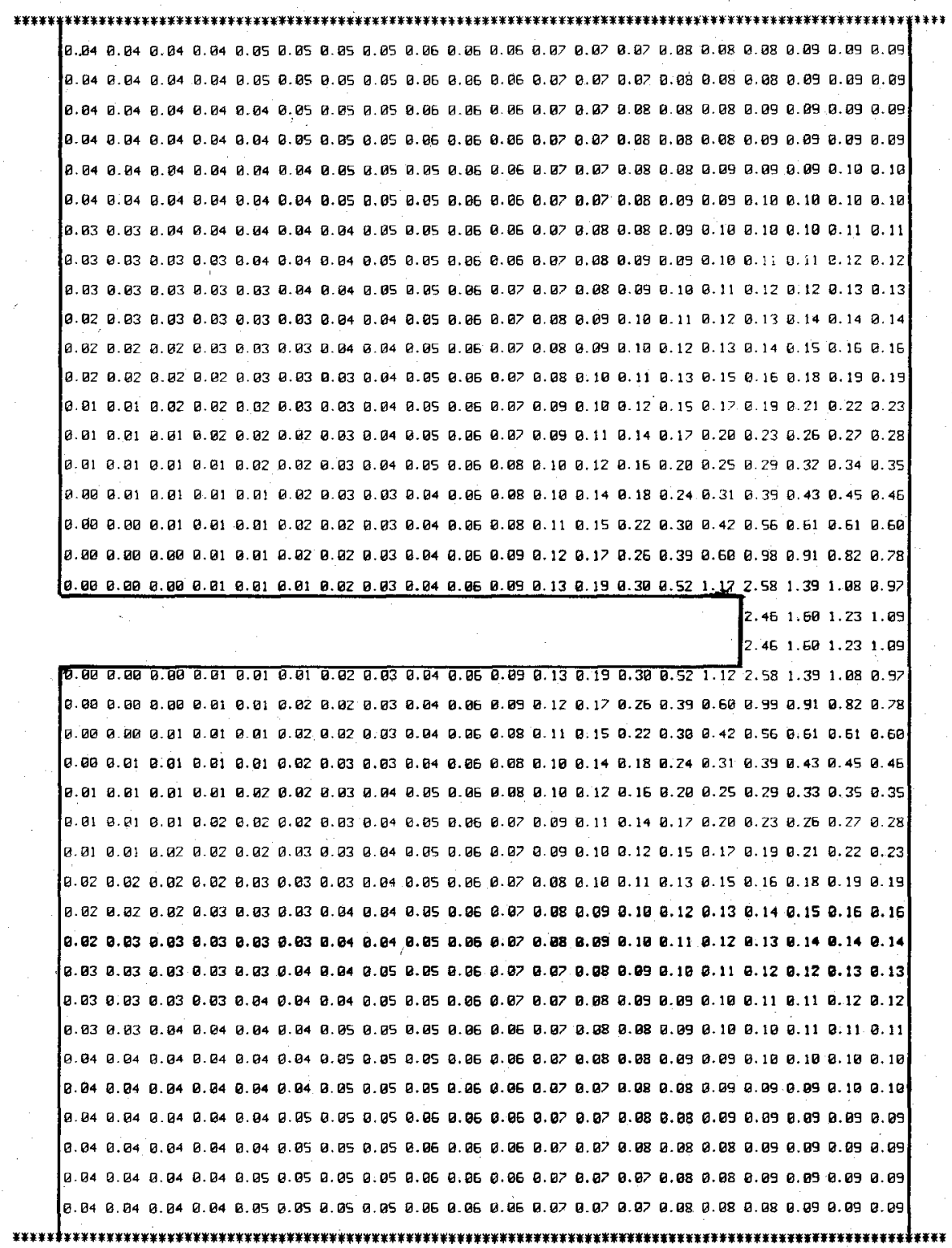

Fig. 12. Comparison of performance of different trim algorithms. (a) Plunge trim. (Figure continued on pp. 1186 and 1187. )

material. Note that the single plunge has a maximum power density at the tip of the plunge which is 2.46 percent compared to a maximum of 0.92 percent for the " $L$ trim" and 1.47 percent for the multiple plunge strategy. Since the trim lengths are considerably different, the fractional power dissipation in the HAZ, specifically $S^{H A Z}$, is more useful for characterizing the trims. These sensitivities are compared in Table I for two cases: 1) assuming that the HAZ has the same sheet resistance of the untrimmed film, and 2) assuming that the sheet resistance of the HAZ is one half of the sheet resistance of the untrimmed material $R_{\square 2}=0.5 R_{\square 1}$. In both cases the width of the HAZ was assumed to be $5 \mu \mathrm{m}$. It can be observed that in both cases with the 200-percent trim, the $L$ trim offers a modest reduction in sensitivity when compared to the plunge schemes and that $S^{H A Z}$ increases significantly for the case $R_{\square 2}=0.5 R_{\square 1}$. Even though the $L$ trim offers a reduction in the sensitivity $S^{H A Z}$ in this example, it is premature to draw a general conclusion about optimal trim strategies.

A comparison of the performance of trimmed bar resistors with other popular geometric structures is of interest. A top-hat resistor with an untrimmed resistance equivalent to the $2: 1$ bar resistor was also plunge trimmed to 200 percent of the initial value as depicted in Fig. 13 along with the relative power densities after trim. $S^{H A Z}$ for this structure was 0.1292 , which is comparable to that obtained for trimming the bar resistors.

The amount of trimming required also significantly affects the $S^{H A Z}$. For example, it can be shown that the $2: 1$ bar resistor trimmed to 150 percent of the initial value 


\section{Fractional Power Dissipated in HAZ $=0.1142$}

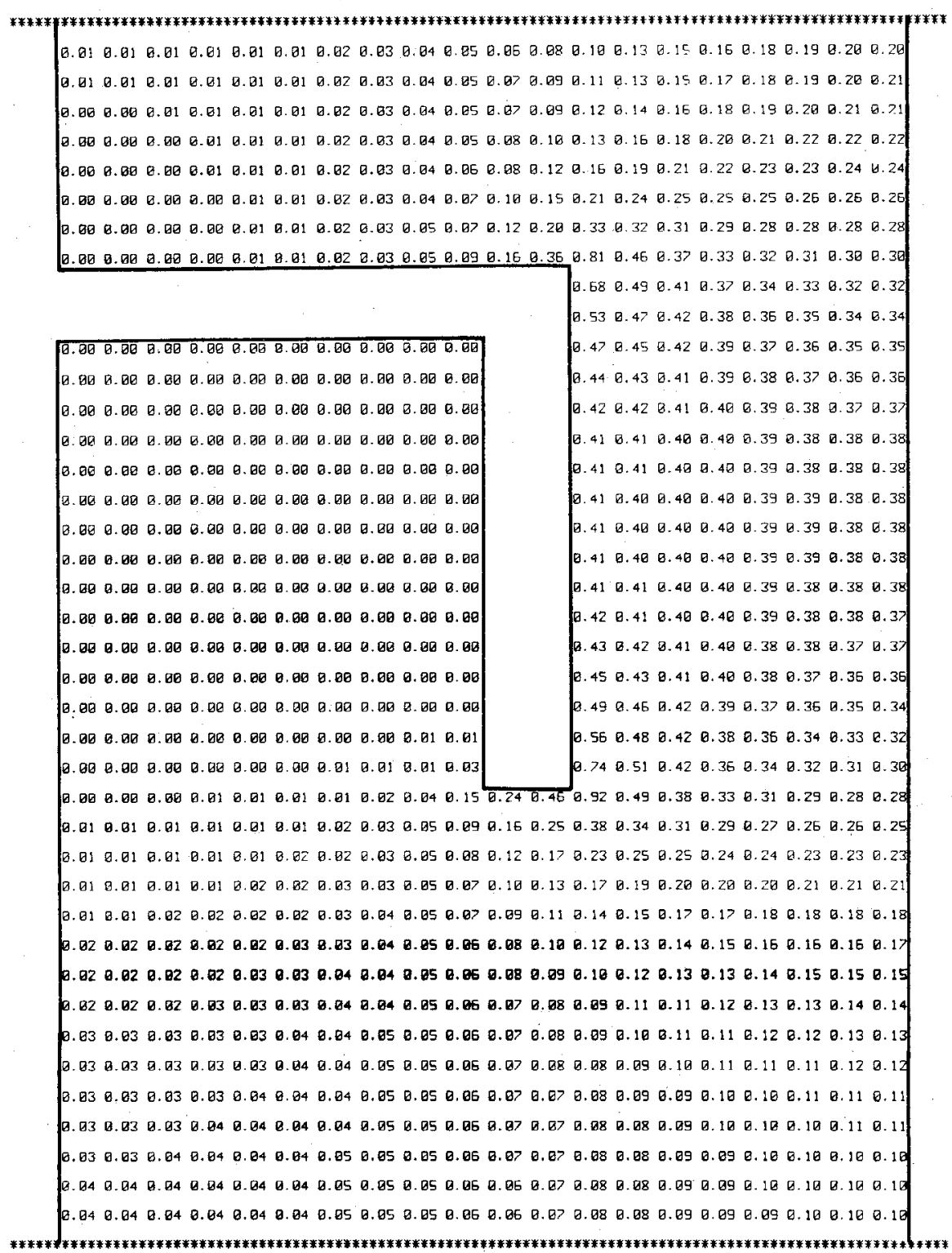

Fig. 12(b) $L$ trim. (Figure continued on p. 1187.)

with a single symmetrical plunge cut results in a value of $S^{H A Z}=0.089$ which is significantly less than experienced for the 200-percent trim.

\section{B. Ratio Accuracy Limitations}

FIRE can also be used to predict ratio accuracy limits. For comparison purposes, a $1: 1$ ratio requirement will be assumed. All resistors are nominal constant width bar resistors. The TCR of the HAZ will be assumed equal to $200 \mathrm{ppm} /{ }^{\circ} \mathrm{C}$ and the TCR of the untrimmed film is assumed to be $100 \mathrm{ppm} /{ }^{\circ} \mathrm{C}$. The nominal sheet resistance of the HAZ, $R_{\square 2}$, will be $0.75 R_{\square 1}$ where $R_{\square 1}$ is the nominal sheet resistance of the untrimmed film.

Two cases will be considered. In case 1 , one resistor $R_{1 A}$ of dimensions $50 \times 100 \mu \mathrm{m}^{2}$ will be fixed and a second $R_{1 B}$ will be $L$ trimmed to equal $R_{1 A}$. The initial value of $R_{1 B}$ will be assumed to be $0.7 R_{1 A} . R_{1 B}$ is assumed intentionally undersized to allow for process variations. In case 2 , it will be assumed that $R_{2 A}$ is $50 \times 100 \mu \mathrm{m}^{2}$ and $R_{2 B}$ is $50 \times 105$ $\mu \mathrm{m}^{2}$ to account for a typical 5-percent mismatch due to processing. $R_{2 A}$ will be initially trimmed with a $25-\mu \mathrm{m}$ symmetric plunge and $R_{2 B}$ will be $L$ trimmed to match $R_{2 A}$. A $5-\mu \mathrm{m}$ laser kerf and a $2.5-\mu \mathrm{m} \mathrm{HAZ}$ will be assumed. The geometric structures are depicted in Fig. 14. The values of $S^{H A Z}$ for the four resistors are shown in Table II along with $\Delta r / r$ as obtained from (31) for the case that $y$ is temperature with $y-y_{0}=100^{\circ} \mathrm{C}$.

The percent error in the ratio in cases 1 and 2 corresponds to 10 and 14 bits, respectively. These represent hard bounds on matching performance with these trim structures and trim algorithms. The difference in resolu- 


\section{Fractional Power Dissipated in $\mathrm{HAZ}=0.1265$}

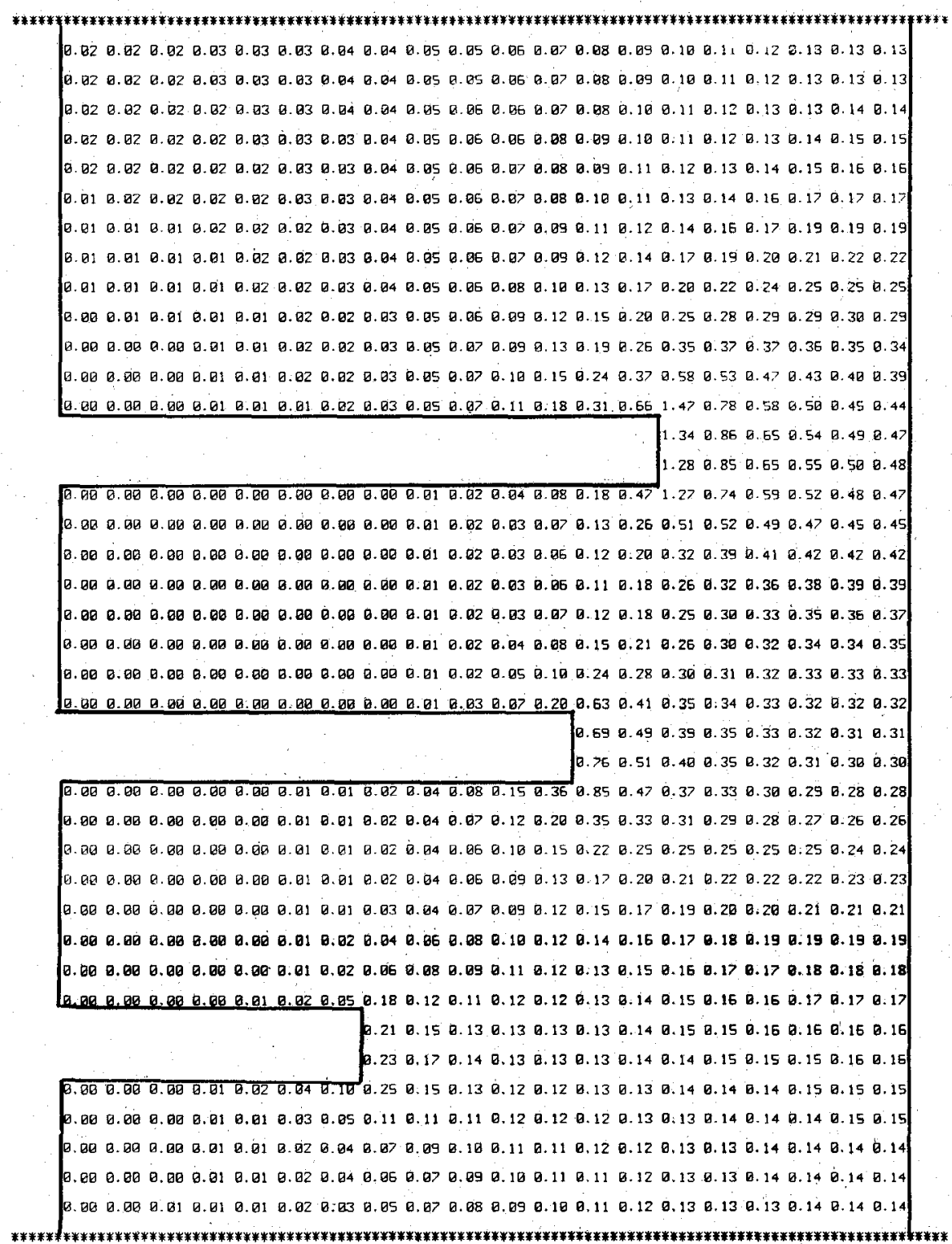

Fig. 12(c) Multiple plunge trim:

TABLE I

Comparison OF Trim STRUCTURES

\begin{tabular}{|c|c|c|c|c|c|c|c|c|}
\hline \multirow[b]{3}{*}{$\begin{array}{l}\text { Number of } \\
\text { Sguares } \\
\text { nu. Wry }\end{array}$} & \multicolumn{4}{|c|}{$s^{\text {HaI }}$} & \multicolumn{4}{|c|}{$\begin{array}{l}\text { Pmax,density } 1 \% \text { of total power } \\
\text { in square/area of squarel }\end{array}$} \\
\hline & \multicolumn{2}{|c|}{$\mathbf{R}_{\mathrm{Dhat}}=\mathbf{R}_{\mathrm{ofil}}$} & \multicolumn{2}{|c|}{$R_{\text {ohaz }}=0.5 R_{\text {film }}$} & \multicolumn{2}{|c|}{$R_{\text {ahaz }}=R_{\text {ofilm }}$} & \multicolumn{2}{|c|}{$R_{\text {ohgz }}=0.5 R_{\text {ofilm }}$} \\
\hline & 20.40 & $60 \cdot 120$ & $20 \cdot 40$ & $60 \cdot 120$ & $20 \cdot 40$ & 60.120 & $20-40$ & $60-120$ \\
\hline $\begin{array}{l}\text { Single } \\
\text { Plunge }\end{array}$ & 0.1516 & 0.1548 & 0.23390 & 0.23657 & 2.4651 & 2.673 & 3.769 & 4.063 \\
\hline L-Plunge & 0.11429 & 0.11605 & 0.19288 & 0.19539 & 0.916 & 0.841 & 0.978 & 1.044 \\
\hline $\begin{array}{l}\text { Multiple } \\
\text { Plunge }\end{array}$ & 0.12646 & 0.13053 & 0.19013 & 0.1955 & 1.470 & 1.468 & 2.102 & 2.301 \\
\hline
\end{tabular}

tion attainable for both trimming algorithms as determined by $S^{H A Z}$ is very significant. Notice that the improvement in accuracy in case 2 was obtained by matching the values of $S^{H A Z}$ even though the sensitivity $S^{H A Z}$ of $R_{A}$ has increased.

\section{CONCLUSIONS}

A method of characterizing the performance of trimmed film resistors has been presented. This method has been implemented in FIRE, a computer program that can analyze arbitrarily shaped film resistors with arbitrary sheet resistance. A figure of merit, $S^{H A Z}$, which is useful for characterizing any trimmed film structure is proposed and readily calculated from the power-density profile of a resistor. 


\section{Fractional Power Dissipated in HAZ $=0.1292$}

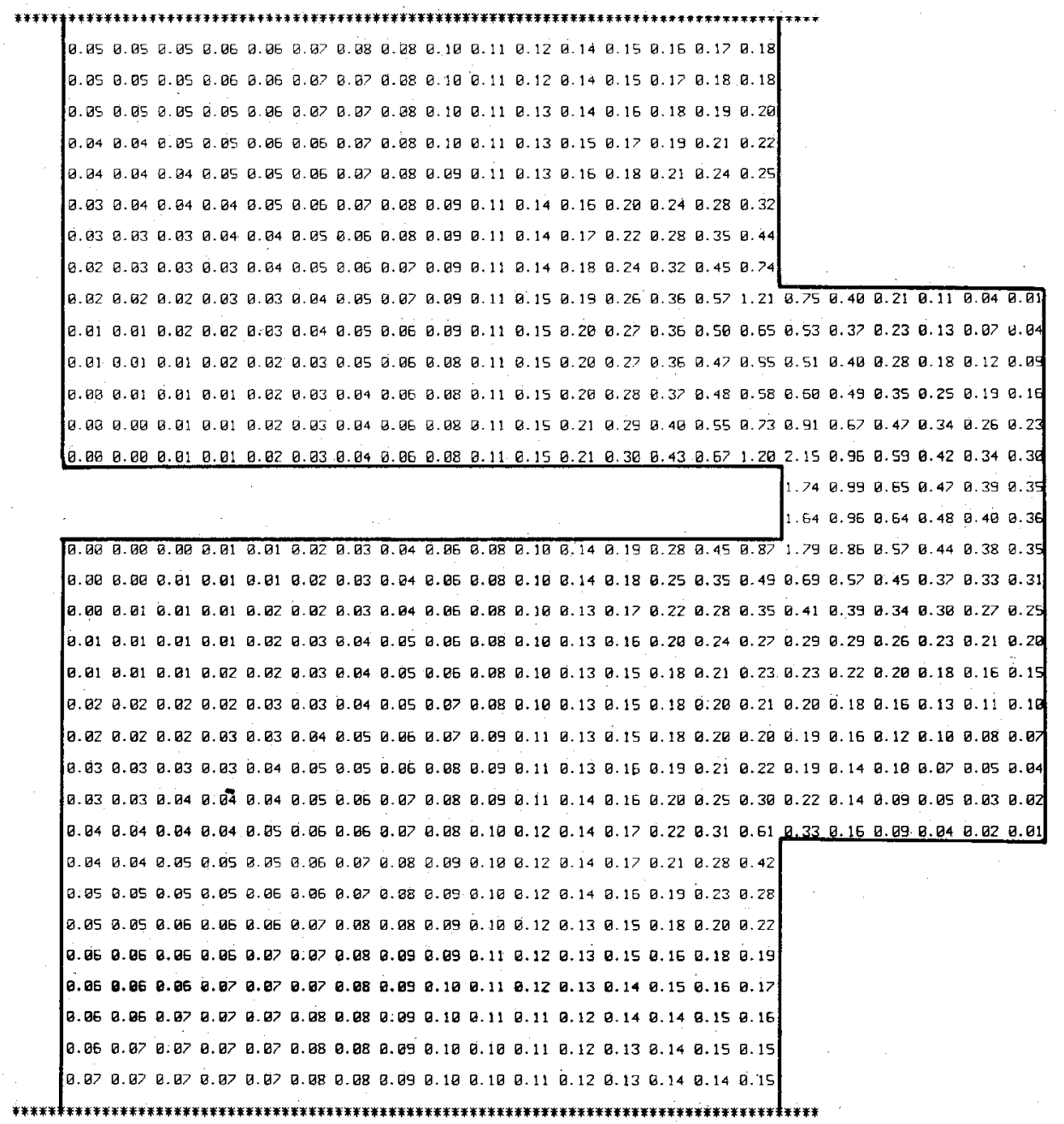

Fig. 13. Performance of plunge-trimmed top-hat resistor.

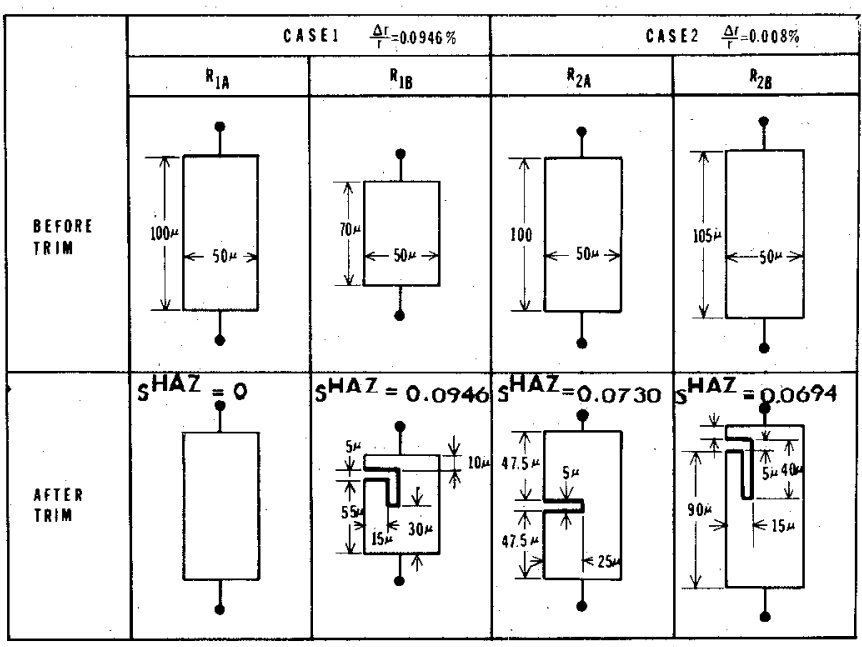

Fig. 14. Ratio accuracy of trimmed film structures.

Simple closed-form expressions for absolute resistor variations and matching performance for arbitrary trimmed films have been presented. These expressions are useful for determining hard bounds on the performance
TABLE II

Matching Performance of the Trimmed Film STRUCTURE OF FIG. 14

\begin{tabular}{|c|c|c|c|}
\hline & $\begin{array}{c}\mathrm{S}^{\mathrm{HAZ}} \\
(\%)\end{array}$ & $\begin{array}{l}\frac{\Delta r}{\tau} \\
(\%)\end{array}$ & $\begin{array}{l}\text { Resolution } \\
\text { (bits) }\end{array}$ \\
\hline $\mathrm{R}_{1 \mathrm{~A}}$ & 0 & \multirow[b]{2}{*}{0.0936} & \multirow[b]{2}{*}{10} \\
\hline $\mathrm{R}_{1 \mathrm{~B}}$ & 0.0946 & & \\
\hline $\mathrm{R}_{2 \mathrm{~A}}$ & 0.0730 & \multirow{2}{*}{-0.0080} & \multirow{2}{*}{14} \\
\hline$R_{2 B}$ & 0.0649 & & \\
\hline
\end{tabular}

potential of a given film structure and trimming strategy.

The performance of several different commonly used film structures was analyzed. These results showed that only modest differences in performance are experienced for changing from plunge to $L$-trimming strategies in bar resistors. The performance of a plunge-trimmed top hat was shown to be comparable to that of the trimmed bar resistors.

Finally, although the performance of the commonly used film structures and trimming algorithms were all seen to be comparable, it can be shown that some structures 
and/or trimming strategies can offer significant improvements over that attainable with the popular structures. These low-sensitivity structures are under investigation and will be reported in the near future.

\section{ACKNOWLEDGMENT}

Technical collaborations with W. Dietrich are gratefully acknowledged.

\section{REFERENCES}

[1] R. W. Berry, P. M. Hall, and T. H. Murray, Thin Film Technology. Princeton, NJ: Van Nostrand Reinhold, 1968.

[2] E. J. Swenson, "The evolution of laser processing in semiconductor manufacturing," in Proc. 26th Midwest Symp. Circuits Syst. (Puebla, Mexico), Aug. 15-16, 1983, p. 212.

[3] R. Wagner, "IC design for laser trimming," in Proc. 26th Midwest Symp. Circuits Syst. (Puebla, Mexico), Aug. 15-16, 1983, pp. 223-226.

[4] A. Kestenbaum, "Trim characteristics of tantalum nitride resistors on silicon," in Proc. IEEE Int. Symp. Circuits Syst. (Montreal, Canada), May 7-10, 1984, pp. 1198-1201.

[5] D. V. Smart, "Considerations of laser material interactions for the trimming of thin film resistors," in Proc. IEEE Int. Symp. Circuits Syst. (Montreal, Canada), May 7-10, 1984, pp. 1185-1188.

[6] R. Dow, M. Mauck, T. Richardson, and E. Swenson, "Reducing post-trim drift of thin-film resistors by optimizing YAG laser output characteristics," IEEE Trans. Components, Hybrid, Manuf. Tech., vol. CHMT-1, pp. 392-397, Dec. 1978.

[7] K. J. Binns and P. J. Lawrenson, Analysis and Computation of Electric and Magnetic Field Problems. Oxford, England: Pergamon, 1973.

[8] L. M. Vallese, "Incremental versus adjoint models for network sensitivity analysis," IEEE Trans. Circuits Syst., vol. CAS-21, pp. 46-49, Jan. 1974.

[9] D. W. Walter, "Computer simulation of resistor trimming," in Proc. 23rd Electron: Components Conf. (Washington, DC), 1973 pp. $56-62$.

[10] P. L. Moran and C. K. Maiti, "A program to predict the resistance of trimmed film resistors," Electrocomponent Sci. Technol., vol. 3, pp. 153-164, 1976.

[11] A. C. Van der Woerd, J. P. Van Lammeren, R. J. H. Hanse, and R. H. Van Beynhem, "Calculation of the resistance value of lasertrimmable planar resistors in an interactive mask-layout system," IEEE J. Solid-State Circuits, vol. SC-10, pp. 532-537, Aug. 1984

[12] A. J. Walton, R. J. Holwill, and J. M. Robertson, "Numerical simulation of resistive interconnects for integrated circuits," IEEE. J. Solid-State Circuits, vol. SC-20, pp. 1252-1258, Dec. 1985.

[13] M. Horowitz and R. W. Dutton, "Resistance extraction from mask layout data," IEEE Trans, Computer-Aided Des., vol. CAD-2, pp. $145-150$, July 1983.

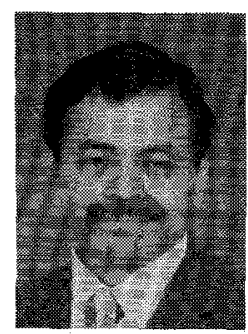

Jaime Ramírez-Angulo (M'76) was born in Mexico City, Mexico, on February 18, 1952. He received a degree in communications and electronic engineering from the National Polytechnic Institute (IPN) of Mexico, Mexico City, the M.S.E.E. degree from the Center for Research and Advanced Studies of Mexico (CINVESTAVIPN), Mexico City, and the Doctor of Engineering degree from the University of Stuttgart, Stuttgart, West Germany, in 1974, 1976, and 1982 , respectively.

He received fellowships from the National Council for Science and Technology (CONACYT), Mexico, and from the German Academic Exchange Service (DAAD), West Germany, during his graduate studies: From September 1982 to June 1984 he worked at the National Institute for Astrophysics, Optics and Electronics (INAOE), Puebla, Mexico, as a
Researcher in the Electronics Department. In July 1984 he joined the Electrical Engineering Department at Texas A\&M University, College Station, where he currently holds the position of Assistant Professor. Hlis research interests include the design of acoustic surface wave devices, hybrid circuits and thin-film technology, statistical circuit design, analog microelectronic circuits, and computer-aided design.

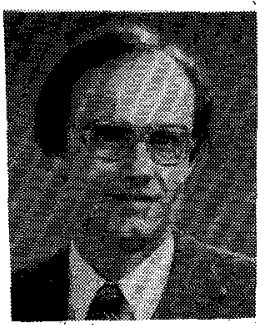

Randall L. Geiger ('S'75-M'77-SM'82) was born in Lexington, NE, on May 17, 1949. He received the B.S. degree in electrical engineering and the M.S. degree in mathematics from the University of Nebraska, Lincoln, in 1972 and 1973, respectively. He received the Ph.D. degree in electrical engineering from Colorado State University, Fort Collins, in 1977.

He currently holds the rank of Professor in the Department of Electrical Engineering at Texas A\&M University, College Station. $\mathrm{He}$ is $\mathrm{co}^{-}$ founder and past president (1982-1985) of the World Instruments Inc., a firm specializing in microprocessor-based instrumentation.

He served as Conference Chairman at the 1983 UGIM Conference, has been a member of the Midwest Symposium Steering Committee since 1980, and is a Registered Professional Engineer in the State of Texas. He was an Associate Editor of the IEEE TRANSACTIONS ON CIRCUITS AND SYSTEMS from 1983 to 1985 , and is currently a Member of the Administrative Committee of the IEEE Circuits and Systems Society and the Circuits and Systems Society Editor of the IEEE Circuits and Devices Magazine. He received the Myril B. Reed Best Paper Award at the 1981 Midwest Symposium on Circuits and Systems for presentation of a paper titled "Switched Resistor Filters."

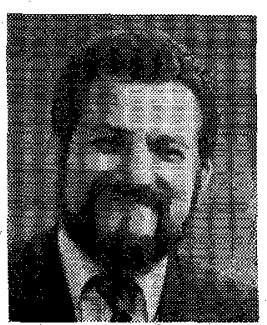

Edgar Sánchez-Sinencio (S'72-M'74-SM'83) was born in Mexico City, Mexico, on October 27, 1944. He received the degree in communications and electronic engineering (Professional degree) from the National Polytechnic Insitute of Mexico, Mexico City, the M.S.E.E. degree from Stanford University, Stanford, $\mathrm{CA}$, and the Ph.D. degree from the University of Illinois at ChampaignUrbana, in 1966, 1970, and 1973, respectively.

During his graduate studies he was awarded with fellowships from the United Nations Eclucational, Scientific, and Cultural Organization, the Mexican Atomic Energy Commission, and the Consejo Nacional de Ciencia y Tecnologia of Mexico. From January 1965 to March 1967 he worked with the Mexican Atomic Energy. Commission as a Design Engineer. In April 1967 he joined the Petroleum Institute of Mexico, where he was associated with the design of instrumentation equipment until August 1967. He worked as a Research Assistant at the Coordinated Science Laboratory, University of Illinois, from September 1971 to August 1973. In 1974 he held an industrial Post-Doctoral position with the Central Research Laboratories, Nippon Electric Company, Ltd., Kawasaki, Japan. From 1976 to 1983 he was the Head of the Department of Electronics at the Instituto National de Astrofisica, Optica y Electronica (INAOE), Puebla, Mexico. He was a Visiting Professor in the Department of Electrical Engineering at Texas A\&M University, College Station, during the academic years of 1979-1980 and 1983-1984, where he is currently a Professor. His present interests are in the area of active filter design, solid-state circuits, and computer-aided circuit design.

Dr. Sánchez-Sinencio was the General Chairman of the 1983 26th Midwest Symposium on Circuits and Systems, and is currently serving as an Associate Editor of News and Events for the IEEE Circuits and Devices Magazine. He is also an Associate Editor for the IEEE TransaCTIONS ON CiRcuits AND SYSTEMS. He is the co-author of the book Switched Capacitor Circuits (Van Nostrand-Reinhold, 1984). 